\title{
Contrastación de un modelo de decisión prospectiva de la sustentabilidad
}

\author{
Francisco Javier Rosas-Ferruzca ${ }^{1, *}$, Francisco Rubén Sandoval-Vázquez², Cruz García-Lirios ${ }^{3}$ \\ ' UAEMEX, Facultad de Planeación Urbana y Regional. \\ 2 UAEM, Facultad de Psicología. \\ ${ }^{3}$ UAEMEX, Facultad de Ciencias del Comportamiento.
}

\begin{abstract}
Resumen
El proceso que va de las decisiones prospectivas (orientadas a riesgos de máximas ganancias respecto a beneficios mínimos seguros) a las acciones deliberadas (orientadas por evitación de fracasos con base en la sistematización de errores y aciertos) es abordado en el presente trabajo a fin de poder contrastar un modelo para el estudio de la gobernanza universitaria como una institución decisora y planificadora de la sustentabilidad. Se llevó a cabo la contrastación en tres fases de la hipótesis nula relativa a las diferencias significativas entre dimensiones teóricas y modelos estructurales. En la primera, se documentó, sintetizó y comparó la información reportada en la literatura mediante la técnica Delphi. En la segunda se revisaron los estudios y los instrumentos seleccionados en la primera fase con la finalidad de delimitar el número de ítems en función de dimensiones y propiedades psicométricas. En la tercera fase se llevaron a cabo dos estudios no experimentales: uno exploratorio en el que se encontraron dos factores y otro confirmatorio en el que ambos factores se asociaron espuriamente sugiriendo la inclusión de otros factores en torno a la decisión prospectiva de una selección no probabilística de 258 estudiantes. Ambos factores reflejaron las razones y las acciones frugales, pero tales hallazgos sólo son aplicables a la muestra ya que el tipo de estudio, muestreo y análisis estadísticos sugieren el contraste del modelo en otros escenarios para incrementar el porcentaje de la varianza explicada.
\end{abstract}

Palabras Clave: Gobernanza universitaria, sustentabilidad, decisión prospectiva. motivación, comportamiento proambiental.

\section{Testing of a prospective sustainability decision model}

The process that goes from the prospective decisions (oriented to risks of maximum gains with respect to minimum insurance benefits) to the deliberate actions (oriented by avoidance of failures based on the systematization of errors and successes) is addressed in the present work to In order to be able to contrast a model for the study of university governance as a decision-making and planning institution for sustainability. The three phases of the null hypothesis regarding the significant differences between theoretical dimensions and structural models were carried out. In the first, the information reported in the literature was documented, synthesized and compared using the Delphi technique. In the second, the studies and instruments selected in the first phase were reviewed in order to delimit the number of items according to dimensions and psychometric properties. In the third phase, two non-experimental studies were carried out: an exploratory one in which two factors were found and a confirmatory one in which both factors were associated spuriously suggesting the inclusion of other factors around the prospective decision of a non-probabilistic selection of 258 students. Both factors reflected the reasons and the frugal actions, but such findings are only applicable to the sample since the type of study, sampling and statistical analysis suggest the contrast of the model in other scenarios to increase the percentage of the variance explained.

Keywords: University governance, water sustainability, prospective decision. motivation, pro-environmental behavior. 


\section{INTRODUCCIÓN}

A menudo, el análisis de la influencia de los organismos internacionales sobre las instituciones públicas se lleva a cabo con la selección, instrumentación y evaluación de estándares y protocolos de sustentabilidad y gobernanza, pero en un sentido diferente, el presente trabajo contrasta los modelos y los instrumentos que miden la decisión de optimización e innovación retrospectiva y prospectiva de recursos y capacidades para establecer un instrumento resultante.

La gobernanza universitaria es entendida como: "la forma en que se ejerce el poder del gobierno que determina, a su vez, la toma de decisiones" (Virgili, Ganga y Figueroa, 2015: p. 197). Es así como en los estudios de la sustentabilidad, la gobernanza universitaria es un marco institucional y normativo que transfiere los lineamientos del Estado a las acciones pro ecológicas de las universidades y los comportamientos proambientales de estudiantes (Carreón, 2013).

A su vez los ejes rectores educativos son derivados de las Cumbres de la Tierra acerca de la reducción de la huella ambiental en general y la huella hídrica en particular. Por consiguiente, la categoría de gobernanza universitaria parece estar posicionada en un contexto de deseabilidad social o decisiones acordes a los lineamientos internacionales sobre los requerimientos locales o académicos (Bustos, Quintero y García, 2017).

En tal sentido es que resulta de particular importancia: 1) observar los efectos de los protocolos internacionales, estatales y académicos sobre la participación estudiantil en materia de reducción de la huella ambiental; 2) medir indicadores de reducción de la huella hídrica como son los casos de los motivos y las habilidades de austeridad; 3) analizar las implicaciones de los factores e indicadores con respecto a la toma de decisiones personales y en relación con la gobernanza universitaria.

El objetivo del presente trabajo es contrastar la hipótesis nula de diferencias significativas entre dimensiones teóricas respecto a un modelo de ecuaciones estructurales de la gobernanza universitaria en materia de sustentabilidad.

\section{Gobernanza universitaria y decisión prospectiva}

La decisión prospectiva, de acuerdo con los propósitos del presente trabajo alude a una racionalidad e irracionalidad, deliberada y emotiva, planificada e improvisada, así como sistemática y emergente que el individuo desarrolla con base en el procesamiento de información (Quintero et al., 2018).
De esta manera, en el caso de las noticias y estrategias relativas a la reducción de la huella hídrica, principalmente de la difusión de la disponibilidad, costo y reutilización del agua como estrategias para alcanzar las exigencias de prácticas sustentables y transparencia informativa, la decisión prospectiva será un factor esencial para establecer el incumplimiento o alcance de los lineamientos institucionales ya que es la comunidad académica la que lleva a cabo las pautas y sistematiza la observación de su debido proceso (García, 2018a).

La relación que guarda el institucionalismo formal y la informalidad gestora de las universidades con respecto a las decisiones prospectivas de sus estudiantes es de suma importancia para entender la diseminación de los lineamientos internacionales en las estrategias y programas académicos sobre el aprendizaje educativo de la conservación de los recursos naturales en general y de los recursos hídricos en particular (Carreón, 2016).

Es sabido que la Agenda 21 y la Cumbre de Rio 92 y 2002 fueron determinantes de los programas de reducción de la huella hídrica en las universidades públicas a través de la educación ambiental en primera instancia y la educación para la sustentabilidad en una versión más reciente del institucionalismo académico (García, Bustos y Montero, 2012).

Sin embargo, la emergencia de la gobernanza universitaria como parte de las políticas públicas de evaluación, acreditación y certificación de la calidad de los procesos y los productos académicos fue un hito en la relación entre autoridades universitarias con respecto a la participación ambiental de las comunidades académicas y estudiantiles (García et al., 2017).

En el nuevo estado rector la gestión sustituyó a la administración pública y las universidades se vieron obligadas a adoptar protocolos de transparencia y rendición de cuentas a las instancias observadoras de los derechos humanos entre los que se encuentran los de tercera generación alusivos a un medio ambiente saludable y con la calidad de sus servicios necesaria para el desarrollo endógeno o local (Sandoval, Bustos y García, 2018).

Sin embargo, muy pronto los protocolos internacionales de gestión, producción y transferencia del conocimiento para la explicación e intervención de los efectos del cambio climático sobre la salud pública develaron su improcedencia en las decisiones personales de las comunidades académicas y estudiantiles (García, 2018b).

Es el caso de la promoción de estilos de vida de autocuidado saludables que incluyeron: deposición de residuos como 
las colillas de cigarros en contenedores espaciales que fueron saboteados y desplazados por otros contenedores generales de basura; nulos efectos de advertencias de los daños a la salud por el consumo de alcohol o tabaco, así como la inacción ante eventos internacionales o locales que demandaron la puesta en práctica de ciclovías o transporte de cero emisiones, pero que las comunidades estudiantiles han adoptado en su minoría (Rivera, Limón, Sandoval y García, 2018).

Algunos otros programas de educación para la sustentabilidad avanzaron hacia el estudio de las normas, los valores, usos y costumbres que, en tanto tradiciones develaron una adscripción de responsabilidad, valores universales, inteligencia emocional y creencias de las consecuencias de los actos (Juárez, Rivera y Hafkam, 2015).

Sin embargo, en ambas dimensiones: cognitivas-racionales y emocionales-colectivas, se ha puesto de relieve que las instituciones de educación superior (IES), públicas y privadas, han fracasado en su intento de construir y diseminar un conocimiento acorde a los lineamientos internacionales, las políticas nacionales, las estrategias académicas o los programas educativos, así como en relación con la participación civil, académica o estudiantil (García, 2018c).

\section{Gobernanza de la sustentabilidad en universidades públicas}

La gobernanza de la sustentabilidad, en el ámbito de las IES, supone una instrumentación de protocolos de conservación de los recursos, transparencia de costos y beneficios, así como corresponsabilidad en cuanto a negociaciones y acuerdos entre autoridades universitarias y comunidades académicas o educativas (Aguilar, García y Carreón, 2018).

Los marcos teóricos que explican la gobernanza de la sustentabilidad son; 1 ) teoría de las decisiones prospectivas; 2) teoría de la justicia social; 3) teoría del capital humano (García, Carreón y Hernández, 2017).

En un sentido teórico, la propensión a los riesgos emerge como un proceso inverso al de una intención prospectiva. Pues bien, quienes asumen decisiones y conductas de riesgo están animados por escasas probabilidades de éxito, esperando el surgimiento de oportunidades de recompensa o reconocimiento (García, Carreón y Bustos, 2012).

En el caso de la decisión prospectiva, los protocolos pueden reducir o incrementar las probabilidades de éxito o cuando menos disminuir los fracasos, pero en un sentido normativo o valorativo estas probabilidades se invierten ya que una decisión prospectiva será tomada siempre que los protocolos generen más pérdidas que beneficios mínimos (García et al., 2015).
Sin embargo, la decisión prospectiva parece ser más resultado de una idea generalizada de justicia que muy poco tendría que ver con los cálculos, aunque mínimos de balance entre oportunidades y capacidades ya que, el individuo que toma una decisión y con base en ella procederá a efectuar una nueva acción, está guiado por un sentido de propensión al equilibrio entre libertad e igualdad (García et al., 2016).

Es decir, la idea de justicia determinará las decisiones y acciones innovadoras al momento de establecer los costos y los beneficios. Si asume que su iniciativa se desenvuelve en un contexto o escenario de justicia, entonces ubicará sus objetivos, tareas y metas en un sentido positivo y en caso de fracaso o perdidas concluirá que su proceder se contrapone al de la mayoría (Hernández et al., 2017).

En contraste, si se predispone a un escenario de injusticia, entonces terminará por reconocerse que prevalecen fuerzas positivas que determinarán el éxito de un proyecto de conservación del agua, o bien, en caso contrario, se asumirán decisiones y actos del debido proceso del bien personal y social (Carreón, 2013).

La teoría de la justicia demostró las insuficiencias del intuicionismo (ideología que consideraba a las personas selectoras de lo que es bueno y lo que es malo) y de la equidad social (las personas nacen con las mismas oportunidades), considerando; a) toda persona posee igual derecho a la más amplia libertad compatible con una libertad igual para todos: b) las desigualdades son inaceptables a menos que sea razonable esperar que actuarán en beneficio de todos y 3) por lo tanto, las posiciones y oficios deben estar abiertos para todos (Carreón, García y Hernández, 2017).

En el marco del Estado liberal como inmoral e injusto supuso una falsa dicotomía: la libertad versus la igualdad, así como la conciliación de ambos valores materializados en un contrato social el cual evolucionó para fundamentar un Estado con base en una síntesis moral (Carreón, De la Cruz y De los Santos, 2015).

La teoría de la justicia social implica un método de equilibrio reflexivo para el estudio de la inmoralidad del Estado liberal; la percepción de libertad, las creencias en torno a la equidad de oportunidades, los valores de igualdad, la moral que busca el bien social a partir de establecer un punto de equilibrio reflexivo entre los valores de la igualdad y las percepciones de libertad, la cooperación y la participación, la sociedad democrática justa; cooperativa, ordenada y estable, así como la redistribución de la riqueza del Estado liberal para una vida plena (García, Carreón y Hernández, 2014).

Si bien la teoría de la justicia complementa el panorama de la teoría prospectiva, es la teoría del capital humano la 
que terminará por ubicar a las decisiones prospectivas en un proceso racional, deliberado, planificado y sistemático, a la vez coexistente con la normativa emotiva, los valores afectivos y los comportamientos espontáneos el cual no garantiza el éxito, pero si anticipa el fracaso (Limón et al., 2017).

Se trata de un proceso en el que la gobernanza de la sustentabilidad se gesta desde una percepción de oportunidades y una factibilidad de llevar a cabo la acción. Es decir, la gobernanza de la sustentabilidad, desde la teoría del capital humano es un proceso adyacente a las decisiones y acciones prospectivas de presunción de riesgos y acciones probables de fracaso en relación con un éxito mínimo (Sánchez et al., 2017).

Pues bien, la maximización del éxito y la supresión del fracaso parece ser el sello distintivo del capital humano, principalmente el capital intelectual porque se orienta hacia un escenario de exacerbada percepción de capacidades instrumentadas para la eficiencia, la eficacia y la efectividad (Sandoval et al., 2017).

Se trata de un aprendizaje social de habilidades y conocimientos en relación con requerimientos del entorno. En ese sentido, es el capital humano e intelectual un instrumento para alcanzar el equilibrio entre las demandas del entorno y los recursos internos de una organización dedicada a la creación del conocimiento (Carreón, 2016).

La eficiencia supone la instrumentación del aprendizaje social de capacidades en función de la disponibilidad de recursos y las demandas externas a la organización. La eficacia refiere al logro de objetivos y metas con base en la realización de tareas para las que es menester un aprendizaje social acelerado. La efectividad es la diseminación del éxito en las relaciones que establece el individuo portador de un capital intelectual y sus capacidades, habilidades y conocimientos (Bustos, Quintero y García, 2017).

Pues bien, en el caso de la gobernanza de la sustentabilidad se evitarán las decisiones y acciones prospectivas sólo si su capital intelectual está orientado al proyecto o modelo que aspira desarrollar y consolidar (García et al., 2011). Es así como la decisión prospectiva es un instrumento de gestión del conocimiento orientado a la toma de decisiones que permitan el desarrollo sustentable, así como la optimización de los recursos y la innovación de los procesos.

\section{Estudios de la decisión prospectiva y el comportamiento proambiental}

En el marco de la sociedad de la información, la gestión del Estado y la autogestión de la comunidad se han diferenciado en cuanto a objetivos, tareas y metas (García, Juárez y Bustos, 2018).

En este sentido, desde las organizaciones de la sociedad civil se han construido modelos de autogestión que consisten en; 1) el diagnóstico de las representaciones sociales de la ciudadanía indicados por el establecimiento de una agenda pública en materia de sustentabilidad, 2) la difusión informativa en torno a la confianza, el compromiso, el emprendimiento, la innovación y la satisfacción como factores determinantes de las representaciones sociales del Estado y la ciudadanía; 3) la evaluación de la difusión de los factores determinantes de la representación del Estado y la ciudadanía (García et al., 2011).

Los estudios de la decisión prospectiva aluden desde el espíritu empresarial hasta la visión estratégica (véase Tabla 1).

Tabla 1. Estudios de la decisión prospectiva

\begin{tabular}{|lll}
\hline $\mathbf{2 0 1 0}$ & Borjas & Espíritu empresarial \\
\hline $\mathbf{2 0 1 1}$ & Adenike & Compromiso ideológico \\
\hline $\mathbf{2 0 1 2}$ & Hazlina, Mohd y Rohaida & Auto gestión ciudadana \\
\hline $\mathbf{2 0 1 3}$ & Zampetakis y Mostakis & Visión Estratégica \\
\hline $\mathbf{2 0 1 4}$ & García et al., & Propensión al futuro \\
\hline $\mathbf{2 0 1 5}$ & Sandoval et al., & Resiliencia \\
\hline $\mathbf{2 0 1 6}$ & Carreón et al., & Oportunismo esperado \\
\hline $\mathbf{2 0 1 7}$ & Limón et al., & Cooperaticvismo \\
\hline $\mathbf{2 0 1 8}$ & García & Fractalidad \\
\hline
\end{tabular}

Fuente: Elaboración propia

La decisión prospectiva refiere a las iniciativas civiles y las propuestas ciudadanas en torno a la sustentabilidad con la finalidad de integrar tales enmiendas en la agenda política, las políticas de gobierno, los programas de prevención de riesgos y las estrategias orientadas hacia la sustentabilidad (Hernández et al., 2017).

Sin embargo, la construcción de una agenda civil o autogestión social supone la difusión informativa de las demandas y recursos, oportunidades y capacidades, ya que son las redes digitales las que cuestionan la agenda pública, o bien, la fortalecen (García et al., 2017).

Por tanto, la decisión prospectiva alude al uso intensivo de Tecnologías de Información y Comunicación, así como dispositivos electrónicos para el establecimiento de una agenda en materia de sustentabilidad, principalmente en la optimización de recursos y la innovación de procesos (García, Carreón y Hernández, 2017). 
Las expectativas locales ante la globalización, indicada por las utilidades, los riesgos, las oportunidades y las capacidades esperadas, suponen un escenario de ventajas competitivas basadas en los activos intangibles y los capitales intelectuales entre los cuales destacan las expectativas como factores de anticipación a conflictos o desencuentros entre los actores económicos, políticos y sociales (Carreón et al., 2013).

En la baraja de expectativas destacan las utilidades percibidas como factores emergentes en las alianzas estratégicas entre multinacionales, IES y pymes, aunque su relevancia estiba en el equilibrio que suponen las demandas externas y los recursos internos en las organizaciones, su función está en el clima de tareas, los cuales determinarían el clima de innovaciones y el clima de apoyos con orientación hacia la sustentabilidad, indicada por la optimización de los recursos y la innovación de los procesos (Carreón et al., 2014).

Sin embargo, consustancial a las utilidades percibidas, las alianzas estratégicas entre multinacionales, IES y pymes suponen riesgos en la transferencia de conocimiento más que en la gestión o producción de innovaciones para el mercado local. Un aumento en los riesgos no sólo implica una reducción de las utilidades esperadas sino, además implica un incremento en las capacidades ya que, en el clima de tareas donde operan los riesgos éstos se subsanan con empatía, confianza y compromiso (Carreón et al., 2016).

Por consiguiente, el clima de relaciones prevaleciente con respecto al clima de tareas, apoyos o confianzas abre oportunidades en la gestión y producción más que en la transferencia de información ya que, en las redes de conocimiento, los climas de tareas son hegemónicos (Carreón et al., 2017).

Si las oportunidades emergen del clima de apoyos y tareas, entonces las capacidades se distinguirán por su grado de habilidades y conocimientos inherentes al clima de innovaciones. Esto es que las habilidades anteceden a las innovaciones, pero subyacen de las relaciones de confianza y empatía, cuando no del compromiso (García et al., 2012).

En relación con el comportamiento proambiental que alude a acciones racionales, deliberadas, planificadas y sistemáticas a favor de la conservación de los recursos naturales para garantizar el desarrollo de las especies animales y vegetales, actuales y futuras; las decisiones prospectivas son determinantes directos, positivos y significativos.

Corral y Queiróz (2004) propusieron a la variable de propensión al futuro, un rasgo de las decisiones prospectivas, como como indicador del comportamiento para la sustentabilidad. En ese tenor, García (2018b) discutió las implicaciones de la propensión al futuro como un antecedente del consenso y la responsabilidad compartida, rasgos fundamentales de la gobernanza.

Vanegas, Ortega, Bustos y Corral (2018) identificaron dos dimensiones relativas a las expectativas proambiental y antiambientales, siendo la primera determinante del comportamiento para la sustentabilidad, aunque las decisiones prospectivas estarían relacionadas con las expectativas, podrían ser modeladas como variables mediadoras al incrementar o reducir el efecto de las dimensiones proambiental y antiambiental en la conservación de los recursos.

García (2017a) contrastó un modelo en el que demostró el proceso de redes, expectativas, decisiones y acciones, centrando su análisis en las dimensiones de selectividad, computabilidad, accesibilidad, compatibilidad, extensionalidad y acumulatividad informativa, principalmente el rasgo de la extensionalidad que alude a información esperada y significativa para las decisiones futuras estaría ligado a las expectativas proambientales y al comportamiento sustentable, siendo dimensión de propensión al futuro la más vinculante.

Es decir, en el proceso que va de las redes informativas hasta el comportamiento para la sustentabilidad, las expectativas y decisiones parecen mediar la influencia de los sistemas persuasivos o coercitivos de gobernanza sobre las acciones de estudiantes, docentes y administrativos.

Una red informativa orientada hacia la optimización de los recursos (frugalidad) más que hacia la innovación de los procesos (tecnología) supone una composición factorial de valores, normas, motivos y disposiciones orientadas al cuidado del entorno, pero si esta red se distingue por promover la invención de oportunidades y la producción de conocimiento, entonces se orientará hacia la innovación tecnológica.

En ambos escenarios, frugalidad y tecnología, la gobernanza de las instituciones de educación superior parece circunscribir sus objetivos, tareas y metas.

\section{Especificación de un modelo para el estudio de la decisión prospectiva}

El establecimiento de un modelo para el estudio de las expectativas de las consecuencias del proceso globalizador centrado en las alianzas estratégicas entre multinacionales, IES y pymes, las cuales se traducen en oportunidades y capacidades de gestión del conocimiento más que de producción y transferencia (García et al., 2013). 
Sin embargo, en un escenario que se distingue por alto grado de relaciones de empatía, confianza y compromiso más que por el cabal funcionamiento de sus estructuras de gestión, producción y transferencia de conocimiento la gobernanza de la sustentabilidad estará limitada (García et al., 2014).

En relación con la identidad como el denominador común entre los desequilibrios que propician las multinacionales en las IE y pymes, valores y normas locales, las expectativas se centran en un tipo de identidad resultante del clima organizacional más que del contexto en el que se enmarca (García, 2013).

Sin embargo, estudios comparativos entre localidades con y sin alianzas estratégicas de multinacionales con IES y pymes han permitido establecer diferencias significativas, así como la incidencia de los actores económicos y políticos sobre los actores sociales, principalmente en los talentos, activos intangibles y capitales intelectuales (García, 2017).

Los valores, creencias y percepciones relativos a necesidades, expectativas, demandas, oportunidades y recursos disponibles para la gobernanza de la sustentabilidad como determinantes de las actitudes, motivos y conocimientos de emprendimiento configuran la toma de decisión prospectiva.

Los valores, creencias y percepciones determinantes de actitudes, motivos y conocimientos inciden en la intención de sustentabilidad. Los valores, creencias y percepciones son determinantes indirectos de las decisiones prospectivas sustentables a través de actitudes, motivos y conocimientos determinantes de intenciones.

Por consiguiente, la pregunta que guía la presente es: ¿Existirán diferencias significativas entre la especificación teórica y la estructura de las relaciones entre factores e indicadores de la toma de decisión prospectiva?

La hipótesis nula que contesta la pregunta es: Existen diferencias significativas entre la especificación teórica y la estructura de las relaciones entre factores e indicadores a observar en la toma de decisión prospectiva

Por consiguiente, la hipótesis alterna es: No existirán diferencias significativas entre la especificación teórica respecto a la estructura de relaciones entre factores e indicadores de la toma de decisión prospectiva

\section{MÉTODO}

El proceso de contrastación incluyó tres fases.

En la primera, se establecieron los ejes y temas de discusión relativos a la gobernanza universitaria de la sustentabilidad, considerando una revisión de la literatura en repositorios internacionales como Copernicus, Dialnet, Ebsco, Latindex, Publindex, Redalyc y Scielo (véase Tabla 1).

Tabla 2. Descriptivos de la literatura revisada

\begin{tabular}{lccc} 
& Sustentabilidad & Gobernanza & Prospectiva \\
Copernicus & 14 & 10 & 7 \\
\hline Dialnet & 10 & 9 & 5 \\
\hline Ebsco & 8 & 7 & 3 \\
Latindex & 6 & 5 & 2 \\
Publindex & 4 & 3 & 1 \\
Redalyc & 2 & 2 & 1 \\
Scielo & 1 & 1 & 0
\end{tabular}

Fuente: Elaborada con los datos del estudio

A partir de la técnica Delphi, jueces expertos asignaron en tres rondas un valor, considerando: -1 para contenidos desfavorables, 0 para información desvinculada y +1 para datos favorables. La sumatoria de las respuestas se concentró en una matriz para su análisis de contenido, obteniéndose la tabla de las dimensiones de las decisiones prospectivas (véase Tabla 3).

Tabla 3. Descriptivos de las calificaciones de jueces a extractos relativos a sustentabilidad, gobernanza y decisión prospectiva.

\begin{tabular}{|cccccc|}
\hline $\mathbf{E}$ & $\mathbf{M}$ & $\mathbf{D}$ & $\mathbf{X}^{2}$ & $\mathbf{g l}$ & $\mathbf{p}$ \\
\hline e1 & ,762 &, 109 & 12,34 & 12 & $<, 05$ \\
e2 &, 703 &, 182 & 14,25 & 13 & $<, 05$ \\
\hline e3 &, 781 &, 104 & 14,36 & 14 & $<, 05$ \\
e4 &, 704 &, 125 & 12,13 & 12 & $<, 05$ \\
\hline e5 & ,782 &, 129 & 10,32 & 14 & $<, 05$ \\
e6 &, 768 &, 146 & 14,37 & 13 & $<, 05$ \\
\hline
\end{tabular}

$\mathrm{E}=$ Extracto: $(\mathrm{e} 1, \mathrm{e} 3, \mathrm{e})=$ Extractos de hallazgos sobre optimización de recursos; (e2, e4, e6) = Extractos de hallazgos sobre innovación de procesos. $\mathrm{M}=$ Media, $\mathrm{D}=$ Desviación Estándar, $X^{2}=$ chi cuadrada, $\mathrm{gl}=$ Grados de libertad, $p=$ nivel de significancia.

Fuente: Elaborada con los datos del estudio

En la segunda fase, se estableció la confiabilidad y la validez de un instrumento que estimó las decisiones prospectivas para el contraste de hipótesis relativas a las diferencias entre la especificación teórica del modelo y la estructura de sus relaciones entre factores e indicadores. El instrumento fue construido a partir de la Escala de Toma de Decisiones de Luna, Laca y Cedillo (2012), la cual incluye tres dimensiones para cada tres ítems, sumando un total de nueve; la Escala de Decisiones de Autoconfianza de Luna y Laca (2014) que 
incluye 16 ítems en una sola dimensión. Ambas escalas fueron piloteadas en muestras de estudiantes y adaptadas al contexto de la investigación, considerando las propiedades psicométricas de distribución normal, fiabilidad y validez (véase Tabla 4).

Tabla 4. Descriptivos de la Escala de Toma de Decisiones y la Escala de Decisiones de Autoconfianza.

\begin{tabular}{|c|c|c|c|c|c|c|c|}
\hline $\mathbf{R}$ & $\mathbf{M}$ & D & A & F1 & F2 & F3 & F4 \\
\hline r1 & 4,12 & 1,92 & 782 & ,540 & & & \\
\hline r2 & 4,39 & 1,04 & 701 & ,621 & & & \\
\hline r3 & 4,30 & 1,03 & 763 & ,430 & & & \\
\hline r4 & 4,17 & 1,78 & ,703 & ,521 & & & \\
\hline r5 & 4,20 & 1,45 & 781 & ,430 & & & \\
\hline r6 & 4,12 & 1,32 & 793 & ,430 & & & \\
\hline r7 & 4,15 & 1,54 & 704 & & ,531 & & \\
\hline r8 & 4,38 & 1,83 & ,761 & & ,430 & & \\
\hline r9 & 4,01 & 1,72 & 778 & & ,672 & & \\
\hline r10 & 4,32 & 1,32 & 793 & & ,561 & & \\
\hline r11 & 4,31 & 1,50 & 763 & & ,672 & & \\
\hline r12 & 4,56 & 1,43 & ,764 & & 681 & & \\
\hline r13 & 4,89 & 1,59 & 706 & & & 602, & \\
\hline r14 & 4,30 & 1,72 & 743 & & &, 573 & \\
\hline r15 & 4,19 & 1,34 & 704 & & & 640 & \\
\hline r16 & 4,10 & 1,25 & 792 & & & 671 & \\
\hline r17 & 4,16 & 1,20 & 783 & & & ,430 & \\
\hline r18 & 4,32 & 1,31 & ,761 & & &, 540 & \\
\hline r19 & 4,10 & 1,43 & 703 & & & & ,430 \\
\hline r20 & 4,30 & 1,40 & 792 & & & & ,418 \\
\hline r21 & 4,18 & 1,28 & ,763 & & & & ,540 \\
\hline $\mathrm{r} 22$ & 4,30 & 1,93 & ,767 & & & & 672 \\
\hline r23 & 4,10 & 1,30 & 786 & & & & ,561 \\
\hline r24 & 4,01 & 1,25 & 798 & & & & ,480 \\
\hline
\end{tabular}

$\mathrm{R}=$ Reactivo, $\mathrm{M}=$ Media, $\mathrm{D}=$ Desviación Estándar, $\mathrm{A}$ = Alfa quitando el valor del ítem. Método: Componentes Principales, Rotación: Varimax. F1 = Optimización Retrospectiva (22\% de la varianza total explicada y alfa de, $\left.780^{\prime}\right)$, F2 = Optimización Prospectiva $(16 \%$ de la varianza total explicada y alfa de ,790), F3 = Innovación Retrospectiva ( $14 \%$ de la varianza total explicada y alfa de ,775), F4 = Innovación Prospectiva (10\% de la varianza total explicada y alfa de ,788). Todos los ítems se responden con alguna de cinco opciones de van desde $0=$ nada probable" hasta $5=$ "bastante probable".

Fuente: Elaborada con los datos del estudio

A partir de la técnica Delphi, la cual consiste en comparar la información e integrar los datos para establecer los ejes y las trayectorias de relaciones entre los factores esgrimidos en la literatura consultada se elaboró la Escala de Toma de Decisiones Sustentables de Bustos (en prensa), utilizando sólo las subescalas de motivos para la sustentabilidad y las habilidades de optimización de recursos como de la innovación de procesos.
La tercera fase incluyó dos instancias.

En la primera instancia, se realizó un estudio exploratorio, transversal y psicométrico a fin de poder establecer la estructura factorial en un modelo de ecuaciones estructurales, considerando la revisión de los instrumentos y sus propiedades psicométricas en una selección muestral no probabilística de 258 estudiantes, considerando sus prácticas profesionales en multinacionales y pymes orientadas hacia la responsabilidad social corporativa en particular y la gobernanza de la sustentabilidad en general (véase Tabla 5).

Tabla 5. Descriptivos de la Escala de Toma de Decisiones Sustentables.

\begin{tabular}{|cccccccc|}
\hline $\mathbf{R}$ & $\mathbf{M}$ & $\mathbf{D}$ & $\mathbf{A}$ & $\mathbf{F 1}$ & F2 & F3 & F4 \\
r1 & 4,16 &, 90 &, 881 &, 644 & & & \\
r2 & 4,35 &, 83 &, 804 &, 627 & & & \\
r3 & 3,34 &, 96 &, 865 &, 638 & & & \\
r4 & 3,17 &, 72 &, 806 &, 620 & & & \\
r5 & 4,23 &, 41 &, 887 & &, 638 & & \\
r6 & 3,11 &, 30 &, 892 & &, 639 & & \\
r7 & 4,14 &, 56 &, 808 & &, 537 & & \\
r8 & 3,32 &, 84 &, 860 & &, 638 & & \\
r9 & 4,07 &, 73 &, 871 & & &, 679 & \\
r10 & 3,38 &, 38 &, 894 & & &, 569 & \\
r11 & 4,30 &, 54 &, 862 & & &, 678 & \\
r12 & 4,53 &, 41 &, 860 & & &, 687 & \\
r13 & 3,82 &, 50 &, 804 & & & &, 609 \\
\hline r14 & 4,31 &, 78 &, 841 & & & &, 579 \\
\hline r15 & 3,15 &, 39 &, 802 & & & &, 648 \\
\hline r16 & 4,13 &, 26 &, 890 & & & &, 679 \\
\hline
\end{tabular}

$\mathrm{R}=$ Reactivo, $\mathrm{M}=$ Media, $\mathrm{D}=$ Desviación Estándar, $\mathrm{A}$ = Alfa quitando el valor del ítem. Método: Componentes Principales, Rotación: Varimax. F1 = Optimización Retrospectiva $(28 \%$ de la varianza total explicada y alfa de ,809'), F2 = Optimización Prospectiva $(22 \%$ de la varianza total explicada y alfa de ,870), F3 = Innovación Retrospectiva (20\% de la varianza total explicada y alfa de ,860), F4 = Innovación Prospectiva (16\% de la varianza total explicada y alfa de ,890). Todos los ítems se responden con alguna de cinco opciones de van desde $0=$ nada probable" hasta 5 = "bastante probable".

Fuente: Elaborada con los datos del estudio

Se encuestaron a los estudiantes en las instalaciones de la universidad pública. La información se procesó en el Paquete Estadístico para Ciencias Sociales (IBM-SPSSAMOS por su acrónimo en inglés versión 25,0).

Se estimaron los parámetros de distribución normal para cada variable, así como los parámetros de distribución normal para el conjunto de las variables seleccionadas. Se 
calcularon los parámetros de confiabilidad y validez para establecer la consistencia del instrumento y la validez de sus factores, utilizando alfa de Cronbach y análisis factorial exploratorio de ejes principales con rotación promax.

En la segunda instancia se llevó a cabo un estudio no experimental, transversal y confirmatorio con una selección muestral intencional de 95 autoridades, funcionarios y administrativos de una universidad pública del centro de México.

Se utilizó el Inventario de Decisiones Prospectivas de Carreón (en prensa) que es una adaptación de la Escala de Decisiones Prospectivas de Bustos (en prensa) el cual incluye 44 reactivos en torno a las dimensiones de empatía, oportunidad, riesgo, utilidad, facilidad, compromiso, emprendimiento, conocimiento, innovación, uso y satisfacción, aunque sólo se utilizaron 16 reactivos que se consideraron por 3 jueces como los más vinculables a las dimensiones teóricas de las decisiones prospectivas, de justicia y capital humano (véase Tabla 5).

De este modo, el Inventario de Decisiones Prospectivas (IDP-16) incluyó cuatro dimensiones relativas a las decisiones prospectivas. Cada ítem se responde con alguna de cinco opciones relativas a $0=$ "nada probable" hasta 5 $=$ "bastante probable".

Se encuestaron a los participantes en las instalaciones de la universidad pública, previa garantía por escrito de confidencialidad y anonimato, así como la advertencia de que los resultados no afectarían ni negativa ni positivamente su estatus académico o empresarial. La información fue procesada en el Paquete Estadístico para Ciencias Sociales (IBM-SPSS-AMOS versión 25,0). Se calcularon los parámetros de confiabilidad, validez, adecuación, esfericidad, ajuste y residual del modelo con la finalidad de contrastar la hipótesis nula.

Con base en los análisis de las escalas se edificó la versión final la cual a continuación se exponen sus propiedades psicométricas.

\section{RESULTADOS}

La Tabla 6 muestra las propiedades psicométricas del instrumento que mide las decisiones prospectivas en sus dimensiones de optimización de recursos (alfa de 0,781) e innovación de procesos (alfa de 0,773).
Tabla 6. Descriptivos de la toma de decisión prospectiva.

\begin{tabular}{cccccccc}
\hline $\mathbf{R}$ & $\mathbf{M}$ & $\mathbf{D}$ & $\mathbf{S}$ & $\mathbf{C}$ & $\mathbf{A}$ & $\mathbf{F 1}$ & F2 \\
r1 & 3,45 & 1,02 & 1,03 & 1,02 &, 705 & &, 273 \\
r2 & 3,56 & 1,04 & 1,04 & 1,24 &, 761 & &, 521 \\
r3 & 3,92 & 1,05 & 1,05 & 1,37 &, 760 & &, 185 \\
r4 & 3,56 & 1,05 & 1,02 & 1,01 &, 734 & &, 521 \\
r5 & 3,25 & 1,02 & 1,01 & 1,92 &, 730 & &, 205 \\
r7 & 3,05 & 1,01 & 1,05 & 1,46 &, 761 & &, 247 \\
r8 & 3,57 & 1,00 & 1,08 & 1,03 &, 735 &, 204 & \\
r9 & 3,25 & 1,08 & 1,08 & 1,38 &, 752 &, 141 & \\
r10 & 3,06 & 1,09 & 1,09 & 1,29 &, 746 &, 346 & \\
r11 & 3,25 & 1,02 & 1,01 & 1,30 &, 762 &, 296 & \\
r12 & 3,45 & 1,03 & 1,02 & 1,05 &, 703 &, 354 & \\
r13 & 3,26 & 1,04 & 1,03 & 1,26 &, 725 &, 225 & \\
r14 & 3,97 & 1,56 & 1,02 & 1,15 &, 706 &, 349 & \\
r15 & 3,10 & 1,36 & 1,01 & 1,16 &, 738 &, 386 & \\
r16 & 3,27 & 1,28 & 1,06 & 1,49 &, 706 &, 305 & \\
\hline
\end{tabular}

$M=$ Media, $\mathrm{D}=$ Desviación estándar, $\mathrm{S}=$ Sesgo, $\mathrm{C}=$ Curtosis, $\mathrm{A}=$ Alfa quitando el valor del ítem. Método de extracción: ejes principales, rotación promax. Adecuación $y$ esfericidad $\int X^{2}=345,34(21 \mathrm{gl}) \mathrm{p}=0,000 ; \mathrm{KMO}=$ 0,751 J $F 1=$ Optimización de recursos ( $26 \%$ de la varianza explicada y alfa de 0,781$)$, F2 = Innovación de procesos ( $17 \%$ de la varianza total explicada y alfa de 0,773 ). Todos los ítems se responden con alguna de cinco opciones que van desde $0=$ nada probable hasta $5=$ muy probable.

Fuente: Elaborada con los datos del estudio

Una vez establecidos los factores que explican $42 \%$ de la varianza total de los constructos y las covarianzas entre los factores e indicadores seleccionados, se procedió a estimar el modelo estructural (véase Figura 1).

Figura 1. Modelo estructural de trayectorias reflejantes.

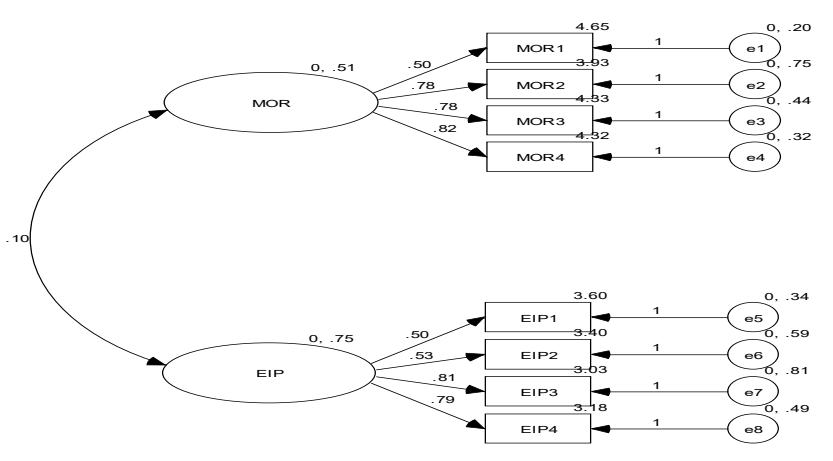

Los parámetros de ajuste y residual $\int X^{2}=13,013$ (19gl) $\mathrm{p}$ $=0,838 ; \mathrm{NFI}=$,963; $\mathrm{RFI}=$,930; $\mathrm{CFI}=$,997; RMSEA = 0,000 sugieren el no rechazo de la hipótesis nula relativa a las diferencias significativas entre la especificación teórica y la estructura de factores e indicadores relativos a la toma de decisión prospectiva en sus factores de optimización de recursos e innovación de procesos. 


\section{DISCUSIÓN}

El aporte del presente artículo al estado de la cuestión radica en la contrastación de un modelo para el estudio de la gobernanza de la sustentabilidad hídrica a partir de la toma de decisiones prospectivas y sus factores de optimización de recursos e innovación de procesos.

En relación con el proceso de contrastación, se demostró un refinamiento de los modelos, hipótesis e instrumentos revisados y probados empíricamente. Esto fue así porque se ajustaron las escalas iniciales a los requerimientos de gobernanza sustentable la institución, permitiendo con ello alcanzar niveles de fiabilidad y varianza explicada superiores a los mínimos indispensables.

Sin embargo, el tipo de estudio no experimental y el tipo de muestreo no probabilístico limitan los resultados del estudio al contexto de la universidad pública y a las muestras, siendo menester recurrir al contraste del modelo en otros escenarios con la finalidad de contrastar la hipótesis nula.

Carreón et al., (2017) demostraron que las políticas de fomento empresarial al incidir sobre las percepciones de optimización de recursos incrementan la sustentabilidad local, pero advierten que el micro-financiamiento no incide en las micros, pequeñas y medianas empresas del mismo modo que el proteccionismo en las grandes empresas ya que, una política generalizada disemina oportunidades que benefician a las grandes empresas como es el caso de la devaluación de la moneda, pero una reducción de los apoyos a micro-empresarios propicia una lógica de decisión prospectiva más que una planificación estratégica.

En el presente trabajo, los factores de las decisiones prospectivas explicaron el $42 \%$ de la varianza total. Ello quiere decir que una incidencia de las políticas de microfinanciamiento en la gobernanza de la sustentabilidad generaría estrategias más que prospectivas a favor de riesgos significativos en relación con ganancias mínimas.

Es decir, el estudio del fenómeno de la gobernanza de la sustentabilidad más bien parece obedecer a un proceso exacerbado de percepción, decisión y acción que estaría siendo acelerado por las políticas de micro-financiamiento, o bien, por las escasas oportunidades del mercado local.

En tal sentido, Sánchez et al., (2017) demostraron que las decisiones prospectivas, entendidas como percepciones, decisiones y acciones orientadas a la maximización de riesgos y beneficios, al no estar influidas por alguna política de crédito o financiamiento, depende de la empatía y la confianza entre acreedores y deudores familiares o sociales que permitiría un margen de decisión y acción más pausado y condicionado por las normas y los valores locales.

En contraste, el presente trabajo ha encontrado que las normas y los valores locales, medidos por la optimización de recursos explicó el $25 \%$ de la varianza total del constructo, siendo proclive o susceptible a complementarse con otros factores, aun y cuando se observen decisiones prospectivas en los micro-empresarios.

García (2018a) descubrió que la empatía, el compromiso y la satisfacción son colaterales a las decisiones prospectivas. Demostró que incluso en ausencia de las políticas de micro-financiamiento, el capital semilla de ex migrantes activo la propensión al futuro, un factor que emerge en comunidades que comparten bienes, saberes y destinos comunes contrarios a la gobernanza de la sustentabilidad.

Pues bien, en el presente estudio se advierte una combinación de los valores y las normas locales, medidas por el factor de la innovación de procesos, con respecto a las acciones deliberadas que suponen un proceso racional y planificado de decisiones, excluyendo las emociones, pero incorporando los saberes comunitarios de las necesidades locales.

En tal sentido es que el fenómeno de la toma de decisiones prospectivas, no obstante que parece estar separado del sentido de comunidad, el arraigo a un entorno y la pertenencia a un espacio compartido es un proceso dual que va de la emotividad a la felicidad y de la racionalidad a la competitividad.

\section{CONCLUSIÓN}

El objetivo del presente trabajo radica en la contrastación de un modelo para el estudio de las decisiones prospectivas, las cuales parecen explicarse desde una aproximación interdisciplinar en cuanto a que se trata de aspectos personales como las decisiones, pero converge en acciones colectivas que serían los casos de las acciones deliberadas y además implica valores y normas locales como es el caso de la justicia percibida.

Sin embargo, el porcentaje explicado de los factores parecen sugerir que pueden ser complementados con otros factores relativos a la empatía, el compromiso, el oportunismo y la satisfacción, aunque la literatura advierte que las políticas de fomento empresarial y el micro-financiamiento son dos detonantes del proceso dual y que, en su ausencia, las decisiones prospectivas se orientan hacia una dimensión social de la cual no se ha desligado del todo. 


\section{REFERENCIAS}

Aguilar, J. A., García, C. y Carreón, J. (2016). Gobernanza de los medios de comunicación: Especificación de un modelo para el estudio el establecimiento de la agenda. México: UPG

Bustos, J. M., Quintero, M. L. y García, C. (2017). Gobernanza de la sustentabilidad local: Índice de mediatización hídrica para la Ciudad de México. Tlatemoani, 24, 143159

Carreón, J. (2013). Discursos en torno a la migración laboral, el retorno y la reinserción social a partir de la identidad grupal en Xilitla, micro-región de la Huasteca Potosina (México). en L. Cano (coord.). Pobreza y desigualdad social. Retos para la reconfiguración de la política social. (pp. 153-174). México: UNAM-ENTS

Carreón, J. (2016). Desarrollo humano: Gobernanza y emprendimiento social. México: UNAM-ENTS

Carreón, J., De la Cruz, P. I. y De los Santos, M. (2015). La administración de temores sociales. Contrastes de un modelo de percepción de la inseguridad en México. Ehquidad, 4, 31-60

Carreón, J., García, C. y Hernández, J. (2017). Las situaciones hídricas, los estilos de vida en las zonas urbanas periféricas y las luchas por el agua. En M. E. Alvarado (coord.). experiencias universitarias de los movimientos sociales y la acción colectiva. Una mirada al pasado y al futuro desde el presente. (pp. 105-128). México: UNAM$\mathrm{CEIICH}$

Carreón, J., García, C., Morales, M. y Rosas, F. (2013). Compromiso laboral en el Trabajo Social del sector salud. Invurnus, 8 (2), 15-23

Carreón, J., Hernández, J. y Quintero, M. L. (2016). Especificación de un modelo de desarrollo local. En D. Del Callejo, Canal, M. E. y Hernández, G. (coord.). orientaciones metodológicas para el estudio del desarrollo. (pp. 149-168). México: Universidad Veracruzana.

Carreón, J., Hernández, J., Bustos, J. M. y García, C. (2017). Políticas de fomento empresarial y sus efectos sobre las percepciones de riesgo en caficultores de Xilitla, san Luis Potosí, centro de México. Poiesis, 32, 33-51

Carreón, J., Hernández, J., Quintero, M. L., García, C. y Mejía, S. (2016). Redes de conocimiento en torno a la complejidad organizacional: aprendizaje de la autoregulación, disipación, adaptabilidad y dinamismo ante los cambios. Prospectiva, 2 (2), 57-70

Carreón, J., Morales, M., Rivera, B., García, C. y Hernández, J. (2014). Emprendedurismo migrante y comerciante: Estado del conocimiento. Tlatemoani, 15, 1-30

Corral, V. y Queiróz, J. (2004). Aproximaciones al estudio de la conducta sustentable. Medio Ambiente y Comportamiento Humano, 5 (1 y 2), 1-26 https://mach. webs.ull.es/PDFS/Vol5_1y2/VOL_5_1y2_a.pdf

García, C. (2013). La inseguridad migratoria en los medios impresos. Reflexiones, 92, 159-173

García, C. (2017a). Contrastación de un modelo de redes decisionales. Mneme, 18 (41), 197-211 https:// periodicos.ufrn.br/mneme/article/view/15391/10609

García, C. (2017b). Los estudios psicológicos del establecimiento de agenda: especificación y discusión de un modelo de identidad conformista versus cooperativista. Nómadas, 50, 1-18

García, C. (2018a). Emprendimiento caficultor en migrantes de la región Huasteca del centro de México. Equidad \& Desarrollo, 30, 119-147

García, C. (2018b). Especificación de un modelo con fuentes de 1987 a 2017 para el estudio de la corresponsabilidad hídrica en una localidad del centro de México. Diálogos de Derecho y Política, 19 (8), 18-38

García, C. (2018c). Interpretación de discursos en torno al subsidio del servicio de abastecimiento hídrico para la comprensión de narrativas tarifarias. Ciencias Sociales, 4 (2), 25-40

García, C., Bustos, J. M. y Montero, M. (2012). Dimensiones de la sustentabilidad hídrica. En J. Carreón, y Hernández, J. 8coord). Psicología de la globalización neoliberal: análisis de la exclusión social, la brecha digital y el desarrollo sustentable. (pp. 117-172). México: Díaz de Santos

García, C., Bustos, J. M., Juárez, M., Rivera, B. L. y limón, G. A. (2017). Expectativas de usuarios del servicio de agua potable en torno al abastecimiento, la calidad y las tarifas en el marco de futuras elecciones en una localidad de la Ciudad de México. Compendium, 4 (7), 35-54

García, C., Carreón, J. y Bustos, J. (2012). Socialización y compromiso laboral en trabajadoras sociales de instituciones públicas y privadas. Humanismo \& Trabajo Social, 11, 177-192

García, C., Carreón, J. y Hernández, J. (2014). La formación profesional del capital humano en la civilización del cambio climático. Revista Internacional de Investigación en Ciencias Sociales, 10 (1), 107-125

García, C., Carreón, J. y Hernández, J. (2017). Hacia un trabajo social del cambio climático y la calidad de los servicios públicos. En Ruíz, M. R. (coord.). Nuevos enfoques de la responsabilidad social. (pp. 227-238). México: UPG

García, C., Carreón, J., Hernández, J. y Aguilar, J. A. (2017). Emprendimiento ciberpolítico para el desarrollo sustentable. En Rivera, R. (coord.). Orientaciones de filosofía de la ciencia. (pp. 110-148). México: UACH

García, C., Carreón, J., Hernández, J. y Bustos, J. (2015). Gobernanza del riesgo desde la percepción de amenazas y el sentido de comunidad. En S. Vázquez., Cid, B. y Montemayor, E. (coord.). Riesgos y Trabajo Social. (pp. 71-94). México: UAT

García, C., Carreón, J., Hernández, J. y Bustos, J. M. (2014). Estructura de las percepciones de riesgo en torno a la escasez y el desabasto de agua global y local. En J. M. Bustos y Flores, L. M. (coord.). Psicología ambiental, análisis de barreras y facilidades psicosociales para la sustentabilidad. (pp. 105-118). México: UNAM-FES Zaragoza

García, C., Carreón, J., Hernández, J. y Bustos, J. M. (2016). Gobernanza del riesgo desde la percepción de amenazas 
y el sentido de la comunidad. En S. Vázquez, Cid, B. G., Montemayor, E. (coord.). Riesgos y trabajo social. (pp. 71-94). México: UAT

García, C., Carreón, J., Hernández, J. y Morales, M. (2014). Contraste de un modelo de compromiso laboral en centros de salud pública. Acta Universitaria, 24 (1), 4859

García, C., Carreón, J., Hernández, J. y Salinas. (2016). Gobernanza de los actores y redes de innovación tecnológica. En M. L. Quintero, Sales, J. y Velázquez, E. B: (coord.). Innovación y tecnología. Retos para su aplicación práctica en las empresas. (pp. 79-94). México: Porrúa-UAEMEX UAP Nezahualcóyotl

García, C., Carreón, J., Hernández, J., Bautista, M. y Méndez, A. (2012). Modelamiento de variables sociopsicorganizacionales a partir de la revisión del estado del arte. Revista de la Facultad de Trabajo Social, 28 (28), 13-60

García, C., Carreón, J., Hernández, J., Bustos, J. M., Valdéz, O., y Vichis, F. J. (2017). Social representations about tamden periurban antrhopocentrism in neighborhood: wáter impact of leaks in local development. En Castellanos, J. A. (coord.). Territorio, turismo, ambiente y tecnología. (pp. 199-205). México: UACH

García, C., Carreón, J., Hernández, J., Bustos, J. y Aguilar, J. (2015). Especificación de un modelo de hipermetropía sociopolítica. Luna Azul, 42, 270-292

García, C., Carreón, J., Hernández, J., Carbajal, C., Quintero, M. L., Sandoval, F. R. y Valdés, O. (2014). Incidencia de las políticas de micro-financiamiento sobre la percepción de emprendimiento caficultor e implicaciones para el Trabajo Social. Equidad, 6, 11-36

García, C., Carreón, J., Hernández, J., Limón, G. A. y Morales, M. L. (2014). Los determinantes de la preocupación hidrológica en Iztapalapa. En J. M. Bustos y Flores, L. M. (coord.). Psicología ambiental, análisis de barreras y facilidades psicosociales para la sustentabilidad. (pp. 93104). México: UNAM-FES Zaragoza

García, C., Carreón, J., Hernández, J., Montero, M. y Bustos, J. M: (2012). Sistemas de complejidad política. Trabajo Social Hoy, 65, 39-48

García, C., Carreón, J., Mecalco, J., Hernández, J., Bautista, M. y Méndez, A. (2013). Sistemas políticos complejos: Implicaciones para la seguridad pública sustentable. Acciones e Investigaciones Sociales, 34,186-216

García, C., Juárez, M. y Bustos, J. M. (2018). Especificación de un modelo para el estudio de la gobernanza local. Sincronía, 22 (73), 459-472

García, C., Valdés, O., Sánchez, R., Elizarraráz, G., Méndez, A. y Hernández, J. (2016). Diferencias entre emprendedores internautas con respecto a empatía, percepciones de riesgo, y uso de aplicaciones tecnológicas. Prospectiva, 2 (1), 69-75

Hernández, J., Carreón, J., García, C., Aguilar, J. A. y López, L. R. (2017). Confiabilidad y validez de un instrumento que mide actitudes hacia la sustentabilidad. En M. L. Quintero, Velázquez, E. B. y Sales, J. (coord.). Enfoques, estrategias y transdisciplina en los estudios de la sustentabilidad. (pp. 95-110). México: Porrúa

Juárez, M. (2010). Sustainability in higher education. An explorative approach on sustainable behavior in two universities. A Doctoral Thesis, Erasmus University Rotterdam, Netherlans.

Juárez, M. (2015). Exploring sustainable behavior structura in higer education. Springer DOI: 10.1007/978-3-31919393-9

Juárez, M., Rivera, J. y Hafkam, W. (2010). An explorative sociopsichological model for determining sustainable behavior: Pilot study in German and Mexicans universities. Journal of Cleaner Production, 18, 686-694

Limón, G. A., Rivera, B. L., Bustos, J. M., Juárez, M. y García, C. (2017). Gobernanza del cambio climático: especificación de un modelo de actitudes hacia los gobiernos del cambio climático. Ciencia Política, 30, 1-31

Luna, A. C. y Laca, F. A. (2014). Patrones de toma de decisiones y autoconfianza en adolescentes bachilleres. Revista de Psicología, 32 (1), 39-55 https://www.redalyc. org/pdf/3378/337831261002.pdf

Luna, A. C., Laca, F. A. y Cedillo, L. I. (2012). Toma de decisiones, estilos de comunicación en el conflicto y comunicación familiar en adolescentes bachilleres. Enseñanza e Investigación en Psicología, 17 (2), 295-311 https://www.redalyc.org/pdf/292/29224159014.pdf

Quintero, M. L., García, C., Rivera, B. L., Sandoval, F. R., Figueroa, O. y Molina, H. D. (2018). Modelo de conciencia para la sustentabilidad. Integración Académica en Psicología, 6 (16), 4-19

Rivera, B. L., Limón, G. A., Sandoval, F. R. y García, C. (2018). Modelo de percepción de riesgos beneficios e intención de voto a favor de ciclovías. Ciencia Política, 33, 1-25

Sánchez, A., Quintero, M. L., Sánchez, R., Fierro, E. y García, C. (2017). Gobernanza del emprendimiento social: especificación de un modelo para el estudio de la innovación local. Nómadas, 51, 1-21

Sandoval, F. J., Bustos, J. M. y García, C. (2018). Gobernanza de la sustentabilidad hídrica local. Gestión de las Personas y Tecnología, 31, 72-87

Sandoval, F. R., Carreón, J., García, C., Quintero, M. L. y Bustos, J. M. (2017). Modelo de los determinantes de la percepción de resiliencia a partir del riesgo y estrés percibidos en relación con la gobernanza de la protección civil. Invurnus, 12 (1), 30-35

Sandoval, F., García, H. y Pérez, A. (2015). Género y resiliencia ante las catástrofes ambientales. Revista Iberoamericana de Producción Académica y Gestión Educativa, 3, 1-20

Vanegas, M. C., Ortega, P., Bustos, J. M. y Corral, V. (2018). Desarrollo de la escala expectativa de comportamiento proambientall de otros con adultos jóvenes mexicanos. Universitas Psychologica, 17 (2), 1-10 https://www. redalyc.org/articulo.oa?id $=64755019007$

Virgili, M., Ganga, F. y Figueroa, K. (2015). Gobernanza universitaria o cogobierno: El caso de la Universidad de Concepción de Chile. Última Década, 42, 187-216 